\title{
Rapid Increase in Clearance of Phenobarbital in Neonates on Extracorporeal Membrane Oxygenation: A Pilot Retrospective Population Pharmacokinetic Analysis
}

\author{
Danica Michaličková, $\mathrm{PhD}^{1}$; Pavla Pokorná, $\mathrm{PhD}^{1-3}$; Dick Tibboel, $\mathrm{PhD}^{3}$; Ondřej Slanař, $\mathrm{PhD}^{1}$; \\ Catherijne A. J. Knibbe, $\mathrm{PhD}^{4,5}$; Elke H. J. Krekels, $\mathrm{PhD}^{4}$
}

\begin{abstract}
Objectives: This study characterizes the changes in the pharmacokinetics of phenobarbital associated with extracorporeal membrane oxygenation treatment in neonates, to illustrate our findings and provide guidance on dosing.

Design: Retrospective pilot population pharmacokinetic analysis. Setting: Neonatal ICU.

Patients: Thirteen critically ill neonates (birth body weight, $3.21 \mathrm{~kg}$ [2.65-3.72 kg]; postnatal age at start of treatment: $2 \mathrm{~d}$ [0-7 d]; gestational age: $38 \mathrm{wk}$ [38-41 wk]) receiving venovenous or venoarterial extracorporeal membrane oxygenation.

Interventions: Phenobarbital administered in a loading dose of $7.5 \mathrm{mg} / \mathrm{kg}(8.5-16 \mathrm{mg} / \mathrm{kg})$ and maintenance dose of $6.9 \mathrm{mg} / \mathrm{kg} / \mathrm{d}$ $(4.5-8.5 \mathrm{mg} / \mathrm{kg} / \mathrm{d})$.
\end{abstract}

Measurements and Main Results: Therapeutic drug monitoring data were available, yielding 5, 31, and 19 phenobarbital concentrations before, during, and after extracorporeal membrane oxygenation, respectively. Population pharmacokinetic analysis was performed using NONMEM 7.3.0 (ICON Development Solutions, Ellicott City, MD). Maturation functions for clearance and volume of distribution were obtained from literature. In a one-compartment model, clearance and volume of distribution for a typical neonate off extracorporeal membrane oxygenation and with a median birth body weight $(3.21 \mathrm{~kg})$ at median postnatal age $(2 \mathrm{~d})$ were $0.0096 \mathrm{~L} / \mathrm{hr}$ (relative SE $=11 \%)$ ) and $2.72 \mathrm{~L}(16 \%)$, respec-

${ }^{1}$ Institute of Pharmacology, First Faculty of Medicine \& General University Hospital, Charles University, Prague, Czech Republic.

${ }^{2}$ Department of Pediatrics, First Faculty of Medicine \& General University Hospital, Charles University, Prague, Czech Republic.

${ }^{3}$ Intensive Care and Department of Pediatric Surgery, Erasmus MC-Sophia Children's Hospital, Rotterdam, The Netherlands.

${ }^{4}$ Division of Systems Biomedicine and Pharmacology, Leiden Academic Centre for Drug Research, Leiden University, Leiden, The Netherlands.

${ }^{5}$ Department of Clinical Pharmacy, St. Antonius Hospital, Nieuwegein, The Netherlands.

Copyright (C) 2020 by the Society of Critical Care Medicine and the World Federation of Pediatric Intensive and Critical Care Societies

DOI: $10.1097 /$ PCC.0000000000002402 tively. During extracorporeal membrane oxygenation, clearance was found to linearly increase with time. Upon decannulation, phenobarbital clearance initially decreased and subsequently increased slowly driven by maturation. Extracorporeal membrane oxygenation-related changes in volume of distribution could not be identified, possibly due to sparse data collection shortly after extracorporeal membrane oxygenation start. According to the model, target attainment is achieved in the first 12 days of extracorporeal membrane oxygenation with a regimen of a loading dose of $20 \mathrm{mg} / \mathrm{kg}$ and a maintenance dose of $4 \mathrm{mg} / \mathrm{kg} / \mathrm{d}$ divided in two doses with an increase of $0.25 \mathrm{mg} / \mathrm{kg}$ every 12 hours during extracorporeal membrane oxygenation treatment.

Conclusions: We found a time-dependent increase in phenobarbital clearance during the first 12 days of extracorporeal membrane oxygenation treatment in neonates, which results in continuously decreasing phenobarbital exposure and increases the risk of therapeutic failure over time. Due to high unexplained variability, frequent and repeated therapeutic drug monitoring should be considered even with the model-derived regimen. (Pediatr Crit Care Med 2020; $21:$ e707-e715)

Key Words: extracorporeal membrane oxygenation; neonates; phenobarbital; population pharmacokinetics

\footnotetext{
xtracorporeal membrane oxygenation (ECMO), also $\checkmark$ known as extracorporeal life support, is a procedure providing life support in severe but potentially reversible cardiorespiratory failure in patients with a predicted chance of long-term survival less than $20 \%$ (1). As of July 2018, over 100,905 patients have been treated with ECMO worldwide, including 40,446 neonates (2). Most neonatal cases treated with ECMO have a primary respiratory diagnosis (viral or bacterial pneumonia and acute respiratory distress syndrome), while the remainder of cases have a primary cardiac diagnosis (cardiopulmonary resuscitation, cardiomyopathy, cardiomyositis, postcardiothoracic surgery) or sepsis (3).
} 
Pharmacotherapy of critically ill pediatric patients on ECMO is complicated because it is influenced by many factors. Generally, volume of distribution is increased between $5 \%$ and $400 \%$ for most drugs, whereas clearance can be decreased or increased compared with patients who are not on ECMO (1). Increase in the volume of distribution principally results from the added blood volume necessary to fill the ECMO circuit (3, 4). Furthermore, a systemic inflammatory response, either related to the patient's clinical condition and/or triggered by the ECMO system, downregulates cytochrome P450 enzymes, may result in reduced clearance of drugs cleared by these enzymes $(3,4)$. Changes in the pharmacokinetics of drugs may also be attributed to the drug adsorption, sequestration, and inactivation by the circuit components (3). The degree of drug adsorption to the ECMO circuit is highly variable and depends on drug properties, circuit type and age, and the clinical state of the patient (4-6). The physicochemical properties of the compound, such as molecular size, lipophilicity, and plasma protein binding, determine the interaction of an individual drug with the ECMO circuit (7). For that reason, the investigation of pharmacokinetics of individual drugs during ECMO is needed to provide optimal dosing recommendations for the patients receiving ECMO.

Phenobarbital is one of most frequently administered anticonvulsive drugs in pediatric patients due to its well-established efficacy, the availability of an injectable dosage form, and its additional beneficial sedative effect (8). Monitoring serum phenobarbital concentrations is often routinely performed to achieve safe and effective individual therapy $(9,10)$. Despite its widespread use, there is insufficient information on the potential impact of ECMO on the pharmacokinetics of phenobarbital. Therefore, the aim of this pilot study is to characterize the pharmacokinetics of phenobarbital in neonatal patients undergoing ECMO. Model-based simulations are used to illustrate the implications our findings may have on dosing in neonates treated with ECMO.

\section{MATERIALS AND METHODS}

\section{Study Design}

Therapeutic drug monitoring (TDM) data which were collected between October 2010 and May 2018 in the neonatal ICU of the General University Hospital in Prague were used for developing a population pharmacokinetic model for phenobarbital in neonatal patients undergoing ECMO therapy. The ECMO therapy was performed by ECMO system consisting of an ECMO pump (Maquet Rotaflow, Rastatt, Germany and for one patient Medos, Stolberg, Germany), coating cannulas (Avalon, Boyle, Ireland or Origen, Austin, TX), and oxygenator (Maquet pediatric Quadrox iD, Rastatt, Germany). The circuit was primed with $250 \mathrm{~mL}$ of blood. Approval of the study was provided by the Ethics Committee of the Department of Ethics, General University Hospital in Prague under the RVproject 64-165/2012. At admission, the patients' parents signed an informed consent wherein they agree that anonymous data can be used for research and publication of the research results.
Patients were included in the study if they were neonates (0-28 d of postnatal age [PNA]), received phenobarbital, and had one or more serum phenobarbital concentration measures while undergoing ECMO. Patients were excluded if they were on dialysis, had severe congenital abnormalities, intracranial hemorrhage, or severe bleeding due to disseminated intravascular coagulopathy.

\section{Phenobarbital Dosing}

Phenobarbital was administered as prescribed by the treating physician. In this study cohort of 13 neonates, two patients received phenobarbital for neuroprotection, while 11 patients received phenobarbital as a sedative drug of whom three were treated for withdrawal symptoms, two had opioid and benzodiazepine tolerance, and the remaining six were treated with phenobarbital as a part of combined analgosedation. Phenobarbital (Phenobarbitalum Natricum; Desitin Arzneimittel GmbH, Hamburg, Germany) was dosed with a median IV loading dose (LD) of $7.5 \mathrm{mg} /$ $\mathrm{kg}$ (interquartile range [IQR], 8.5-16 mg/kg) administered in 15 minutes; another LD could be given if clinically indicated until a maximum total LD of $40 \mathrm{mg} / \mathrm{kg}$ was reached. An IV maintenance dose (MD) of $6.9 \mathrm{mg} / \mathrm{kg}(4.5-8.5 \mathrm{mg} / \mathrm{kg})$ a day was divided in two doses every 12 hours administered in 15 minutes (11). Dose adjustments were based on clinical and/or amplitude-integrated electroencephalography response. The duration of phenobarbital treatment was 134 hours (83-516hr).

\section{Bioanalytical Assay}

Blood samples were taken from the arterial line. Serum was separated by centrifugation $(1,500 \times \mathrm{g}, 15 \mathrm{~min})$ and immediately used for phenobarbital level determination. Total phenobarbital concentrations were measured by fluorescence polarization immunoassay (TDxFLx Phenobarbital Abbott laboratories, Diagnostics Division, Abbott Park, IL) at the biochemical laboratory of the Department of clinical biochemistry and microbiology, General University Hospital, the first Faculty of Medicine of Charles University (12). Fluoresceinlabeled phenobarbital binds an antibody and the emitted light is polarized due to the reduction in freedom of rotation. When phenobarbital is present in the patients' serum samples, it reduces the extent of fluorescence polarization. The test range of the assay was $1.87-61.60 \mathrm{mg} / \mathrm{L}$. Coefficient of variation of intra-assay was less than $7 \%$.

\section{Population Pharmacokinetic Analysis}

The data analysis was performed using NONMEM Version 7.3.0 (ICON Development Solutions, Ellicott City, MD) and PsN v3.4.2 (13, 14) both running under Pirana 2.9.0 (15). R 3.3.2 was used for the visualization of the data and model diagnostics.

Model development was performed in three steps.

1) Development of structural and statistical model: For the structural model, one and two-compartment models were tested to describe the distribution of phenobarbital. First-order clearance of phenobarbital was assumed. Log-normally distributed inter-individual variability terms 
with estimated variance were tested on each pharmacokinetic parameter. Proportional, additive, and combination error models were tested for the residual error model.

2) Covariate analysis: During prolonged ECMO treatment, the impact of maturation, disease progression or clinical recovery, and ECMO treatment on phenobarbital pharmacokinetics cannot be distinguished from each other. Therefore, maturation functions from a previously published model in patients with an overlapping age-range that did not receive ECMO treatment (16) were included in the model a priori. These maturation functions are based on birth body weight (bBW) and PNA for clearance and on actual body weight (BW) for volume of distribution. Since BW measurements during ECMO are not feasible, last BW measured before start of ECMO was used as covariate value for volume of distribution throughout the duration of ECMO.

After incorporation of the maturation functions, the remaining impact of disease progression or clinical recovery and ECMO treatment were evaluated by testing covariates related to the following variables:

- Disease status: Laboratory values, including serum creatinine, serum urea, serum albumin, total bilirubin and direct bilirubin, C-reactive protein, blood $\mathrm{pH}$, aspartate transaminase, and alanine transaminase, as well as urine output, were tested as continuous covariates;

- Concomitant therapy: Use of diuretics, inotropes, and therapeutic hypothermia as well as use of continuous renal replacement therapy were tested as categorical covariates;

- ECMO: On/off ECMO, ECMO modalities (venovenous, venoarterial), and change of ECMO circuit were tested as categorical covariates and duration, speed, flow, time after start and stop of ECMO were tested as continuous covariates.

For all continuous covariates, multiple time-varying measurements were available. A stepwise covariate modeling procedure was performed. Continuous covariates were tested in linear and power functions. Categorical covariates were tested by estimating the parameter value for one category as a fraction of the parameter value for the other category.

For model selection, a decrease in objective function of more than 3.84 points between nested models $(p<0.05)$ was considered statistically significant, assuming a chi-square testdistribution. Additional criteria for model selection were relative SE (RSE) of the estimates of structural model parameters less than $50 \%$, condition number less than 1,000, physiologic plausibility of the obtained parameter values, and absence of bias in goodness-of-fit (GOF) plots.

3) Validation of the final model: To evaluate the robustness of the model and identify potential influential individuals, a jackknife analysis was performed, by excluding one patient from the dataset at a time and reestimating all model parameters in the final model.
The predictive properties of the structural and statistical model were validated using normalized prediction distribution errors (NPDEs). For this, the dataset was simulated 500 times, after which the observed concentrations were compared with the range of simulated values using the NPDE package developed for $\mathrm{R}$ ( $\mathrm{R}$ Foundation for Statistical Computing, Vienna, Austria; http://www.R-project.org) (17).

\section{Model-Derived Dosing Implications}

To illustrate the implications of our findings, simulations were performed with the final population pharmacokinetic model, which included inter-individual variability in model parameters, to evaluate the probability of target attainment. As no target concentration has yet been defined for neonates treated with phenobarbital for sedation and neuroprotection, target concentrations for neonatal seizures (15-40 mg/L) (18) were used. One-thousand simulations for newborns with a bBW of $3.21 \mathrm{~kg}$ and ages of: $0,7,14,21$ days were performed for a $\mathrm{LD}$ of $20 \mathrm{mg} / \mathrm{kg}$ and different MD. For MD, simulations included a dose of $5 \mathrm{mg} / \mathrm{kg} / \mathrm{d}$ for neonates of PNA $=0-14$ days and a dose of $6 \mathrm{mg} / \mathrm{kg} / \mathrm{d}$ for neonates of PNA $=15-28$ days divided in two daily doses, which is recommended by the Dutch National Children's Formulary (18) and a dosing regimen starting with the recommended dose with a time-dependent increase proportional to the increase in clearance in the final model. The latter dosing regimen achieved target concentrations attainment in the simulated individuals. In the simulations, simultaneous start of ECMO and phenobarbital therapy was assumed. A maximum ECMO duration of 12 days was simulated, as a longer duration is not supported by the model.

\section{RESULTS}

\section{Patient Population and Data}

Thirteen patients (seven male and six female) (median [IQR] bBW: $3.21 \mathrm{~kg}$ [2.65-3.72 kg], PNA at start of treatment: $2 \mathrm{~d}$ [0$7 \mathrm{~d}$ ], and GA: $38 \mathrm{wk}$ [38-41 wk]) were included in the analysis. Demographics and treatment details are presented in Table 1.

In total, 55 phenobarbital concentrations (five concentrations before ECMO, 31 during ECMO, and 19 concentrations after ECMO) were included in the analysis. The median number of blood samples per patient was 2 (1-8.5). Phenobarbital levels ranged between 2.8 and $56.4 \mathrm{mg} / \mathrm{L}$. Supplementary Figure 1 (Supplemental Digital Content 1, http://links.lww.com/PCC/ B350) shows the phenobarbital concentrations plotted against time after the first dose.

\section{Population Pharmacokinetic Model}

Observed phenobarbital plasma concentrations were best described by a one-compartment model with log-normally distributed intra-individual variability (IIV) on clearance and volume of distribution. An additive residual error model provided the best description of residual variability.

For the covariate model, it was first confirmed that the covariate relationships obtained from literature to describe the maturation of clearance and volume of distribution of 


\section{TABLE 1. Clinical Characteristics of the Patients Included in This Analysis}

Parameter (Unit)

Value $^{a}$

Age (d) at the start of ECMO treatment

Gestational age (wk)

$2(0-7)$

Birth bodyweight $(\mathrm{kg})$

$38(38-41)$

Body weight $(\mathrm{kg})$ at the start of ECMO treatment

Sex, male/female, $n$ (\%)

Survival, $n$ (\%)

$3.21(2.65-3.72)$

$3.23(2.67-3.72)$

$7 / 6(54 / 46)$

$8(61.5)$

Phenobarbital use

Loading dose $(\mathrm{mg} / \mathrm{kg})$

$7.5(8.5-16)$

Maintenance dose $(\mathrm{mg} / \mathrm{kg} / \mathrm{d})$

$6.9(4.5-8.5)$

Duration of treatment (hr)

$134(83-516)$

Primary indication for ECMO, $n$ (\%)

Respiratory failure

$10(76.9)$

Persistent fetal circulation

Sepsis

$1(7.7)$

$1(7.7)$

Congenital diaphragmatic hernia

Laboratory values at the start of treatment

Creatinine $(\mu \mathrm{mol} / \mathrm{L})$

$61(43-63)$

Urea (mmol/L)

$12(2.3-25)$

Total bilirubin ( $\mu \mathrm{mol} / \mathrm{L})$

Direct bilirubin ( $\mu \mathrm{mol} / \mathrm{L})$

Albumin ( $g / L)$

C-reactive protein ( $\mathrm{mg} / \mathrm{L})$

Aspartate transaminase (IU/L)

Alanine transaminase (IU/L)

$\mathrm{pH}$

Urine output before ECMO start (mL/ $\mathrm{kg} / \mathrm{hr})$

Urine output during ECMO ( $\mathrm{mL} / \mathrm{kg} / \mathrm{hr})$

Urine output after ECMO cessation $(\mathrm{mL} / \mathrm{kg} / \mathrm{hr})$

$58(38-146)$

$4(1-59)$

$31(26-35)$

$14(0-29)$

$1.2(0.70-2.7)$

$0.45(0.23-0.74)$

$7.38(7.35-7.45)$

$5.3(2.9-5.9)$

$6.5(4.8-7.7)$

$6.5(5.3-7.4)$

109 (50-204)

$1(7.7)$

$11(84.6)$

$1(7.7)$

$2(15.4)$

$0.35(0.25-0.45)$

ECMO flow (L/min)

ECMO speed (revolutions/min)

$2,340(2,075-2,620)$

Concomitant treatments, $n$ (\%)

Diuretics

$13(100)$

$13(100)$

1 (9)

Continuous renal replacement therapy

Therapeutic hypothermia phenobarbital, led to accurate predictions of phenobarbital concentrations obtained prior to the start of ECMO. This was indicated by the lack of bias in GOF plots for these samples and by the fact that all conditional weighted residuals values were between -2 and +2 , which is the range where $95 \%$ of the observations are expected to be found.

Inclusion of ECMO as a binary covariate (i.e., on/off) on clearance resulted in a statistically significant improvement of the model fit $(p<0.001)$. Adding time since the start of ECMO in a linear relationship as a covariate on clearance, further improved the fit $(p<0.001)$. The estimation of an exponential increase in clearance over time since start of ECMO did not statistically significantly improve the model fit further, therefore the linear relationship was retained in the model. After inclusion of this covariate relationship, none of the other covariates were statistically significant.

The final parameter estimates are presented in Table 2 and the final model code will be made available in the Drug Disease Model Resources model repository (http://repository.ddmore.eu/). In the final model, clearance and volume of distribution for a typical neonate of the median bBW of $3.21 \mathrm{~kg}$ at the median PNA of 2 days that was off ECMO were $0.0096 \mathrm{~L} / \mathrm{hr}(\operatorname{RsE}=11 \%))$ and $2.72 \mathrm{~L}(16 \%)$, respectively. To illustrate the impact of ECMO on phenobarbital pharmacokinetics: these parameters for this typical neonate 1 day after

\section{TABLE 2. Parameter Estimates of the Final Model}

Parameter (Units) Final Model (Rse \%)

Fixed effects

$\mathrm{CL}(\mathrm{L} / \mathrm{hr})=\mathrm{CLp} \times(1+\theta \mathrm{bBWCL} \times[\mathrm{bBW}-2.59]) \times(1+$ $\theta \mathrm{AGE} \times[\mathrm{AGE}-4.50]) \times\left(1+\theta \mathrm{TE} \times[\text { TE/109]) })^{\text {ECMO_on }}\right.$

CLp (L/hr)

$0.0096(11 \%)$

$\theta b B W C L$

$0.369 \mathrm{FIX}$

$\theta A G E$

$0.0533 \mathrm{FIX}$

$\theta \mathrm{TE}$

$1.09(28 \%)$

$\mathrm{Vd}(\mathrm{L})=\mathrm{Vp} \times(1+\theta \mathrm{BWV} \times[\mathrm{BW}-2.70])$

$\mathrm{Vp}(\mathrm{L})$

$2.72(16 \%)$

ӨBWV

$0.309 \mathrm{FIX}$

Inter-individual variability

CL (\%)

$29.4 \%(26 \%)$

$\operatorname{Vd}(\%)$

$45.3 \%(17 \%)$

Residual variability

Proportional (\%)

$4.41(32 \%)$

$\mathrm{bBW}=$ birth body weight, $\mathrm{BW}=$ body weight, $\mathrm{CL}=$ clearance, $\mathrm{CL} \mathrm{p}=$ population clearance value, ECMO_on = binary parameter indicating whether extracorporeal membrane oxygenation treatment is on (1) or off (0), FIX = fixed, $\mathrm{RSE}=$ relative $\mathrm{SE}$ of the estimate, $\mathrm{Vd}=$ volume of distribution, $\mathrm{Vp}=$ population volume of distribution value, $\theta \mathrm{AGE}=$ increase in clearance per postnatal day, $\theta \mathrm{bBWCL}=$ increase in clearance per kg birthweight, $\theta \mathrm{BWV}=$ increase in volume of distribution per $\mathrm{kg}$ actual bodyweight, $\Theta T E=$ increase in clearance per hr time after extracorporeal membrane oxygenation cannulation (TE).

$\mathrm{ECMO}=$ extracorporeal membrane oxygenation, $\mathrm{IU}=$ international units

a Values are presented as median (interquartile range) unless stated otherwise. 
start of ECMO are $0.011 \mathrm{~L} / \mathrm{hr}$ and $2.72 \mathrm{~L}$, respectively, and after 10 days of ECMO treatment, the parameter values are $0.033 \mathrm{~L} / \mathrm{hr}$ and $2.72 \mathrm{~L}$, respectively.

The model findings regarding the changes of phenobarbital clearance during ECMO are graphically illustrated in Figure 1, in which clearance for a typical individual $(\mathrm{BW}=3.21 \mathrm{~kg}$, PNA at start of ECMO $=2 \mathrm{~d}$ ) through the course of time is depicted. Before and after ECMO, the clearance of phenobarbital increases slowly as the neonates mature. On top of this, clearance increases linearly with time during ECMO. After decannulation, phenobarbital clearance was found to decline rapidly to the values expected based on the age and weight of the neonate (Fig. 1).

Although the number of individuals in our analysis and the number of observations per individual were small, the data are sufficiently informative to support estimation of the model parameters which is indicated by the condition number of $49.42(<1,000)$. The precision of the estimated parameter values is also acceptable, with Rse values around or below $30 \%$. The basic GOF plots in Figure 2 indicate that the final model can describe the data accurately, as the predicted population and predicted individual concentrations are described without bias before, during, and after ECMO treatment.

The distribution of the NPDEs obtained with the model for the dataset has a mean of -0.0799 and variance of 1.034 . Neither of these values are significantly different from the expected values of $0(p=0.53)$ and $1(p=0.56)$, respectively (Supplementary Fig. 2, Supplemental Digital Content 1, http://links.lww.com/ $\mathrm{PCC} / \mathrm{B} 350)$. This indicates that predictions regarding the structural model and the variability in the data are accurate.

Small deviations in parameter values are always to be expected with the exclusion of individuals from the model fit in

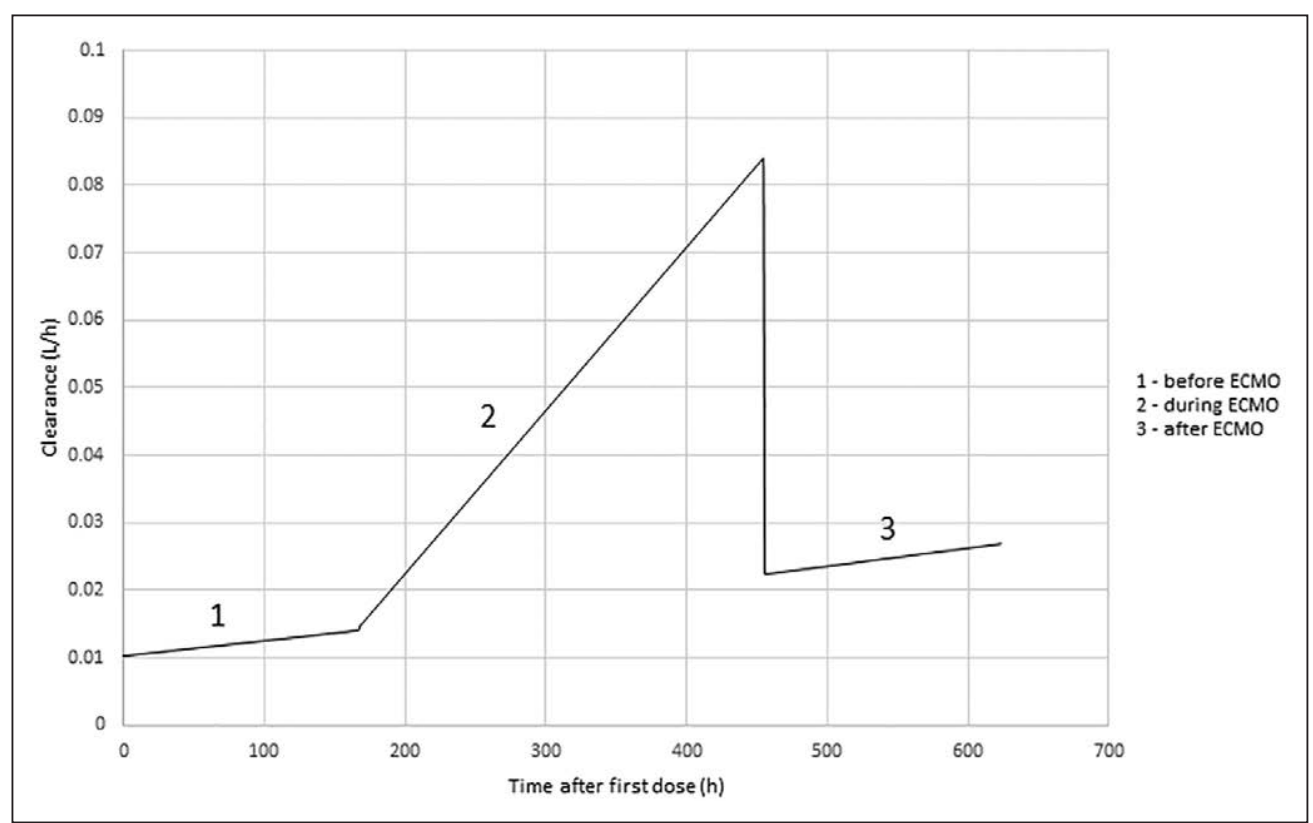

Figure 1. Predicted clearance values for a typical individual (body weight $=3.21 \mathrm{~kg}$, postnatal age $=2 \mathrm{~d}$ at the start of treatment) through the course of time. The figure represents a patient being on phenobarbital therapy: a week before extracorporeal membrane oxygenation (ECMO) initiation (1), then $12 \mathrm{~d}$ on ECMO (2), and a week after ECMO cessation (3). a jackknife procedure, due to differences in number of samples, sampling times and dosing between individuals and due to differences in range or distribution of covariate values that are introduced when removing an individual from a dataset. The structural parameter estimates from the jackknife samples were however all within $\pm 10 \%$ of the estimates obtained in the original model fit. The only exception was noted for the estimate of volume of distribution $(+13 \%)$ when patient 6 was excluded. For the IIV parameters, a maximum difference of $-19.5 \%$ was noted, when patient 1 was excluded. The parameters in the covariate relationship describing the increase in clearance over time after ECMO cannulation from the jackknife samples were all within $\pm 15 \%$ of the covariate parameter of the original dataset, apart from one sample leading to a difference $+22 \%$, which was observed for patient 1 . We did not identify any deviating patient characteristics for patients 1 and 6. As there are overall only small deviations in obtained parameter values with the exclusion of individuals from the dataset, it can be concluded that no individuals with a large influence on estimated parameter values were present.

\section{Model-Derived Dosing Implications}

The simulations of the current dosing regimen (LD of $20 \mathrm{mg} /$ $\mathrm{kg}$ and a $\mathrm{MD}$ of $5 \mathrm{mg} / \mathrm{kg} / \mathrm{d}$ for neonates of PNA $=0-14 \mathrm{~d}$ and $6 \mathrm{mg} / \mathrm{kg} / \mathrm{d}$ for neonates of PNA $=15-28 \mathrm{~d}$ divided in two daily doses) in Figure $3 \boldsymbol{A}$ shows high IIV in pharmacokinetics of phenobarbital during ECMO treatment, as reflected in the wide $95 \%$ prediction interval. Furthermore, the simulations also indicate that the applied recommended MD leads to an increasing number of neonates being under-dosed over time when they are on ECMO treatment.

As clearance is the driver of steady state concentration, and therefore also of $\mathrm{MD}$, the model suggests that optimal dosing will be achieved by a regimen that includes a $\mathrm{MD}$ starting with $4 \mathrm{mg} / \mathrm{kg} / \mathrm{d}$ that is increasing with $0.25 \mathrm{mg} / \mathrm{kg}$ every 12 hours during ECMO treatment. Figure $3 B$ illustrates the simulated concentrationtime profiles for the same individuals with the model-derived dosing regimen. Overall, these results suggest that more neonates will be dosed adequately, and therefore, less dose adjustment will be necessary when this dosing regimen is followed. However, as the $95 \%$ prediction interval of simulated concentrations is still outside of the therapeutic range, TDM is still warranted. When ECMO stops, phenobarbital clearance returns back to the value that is 

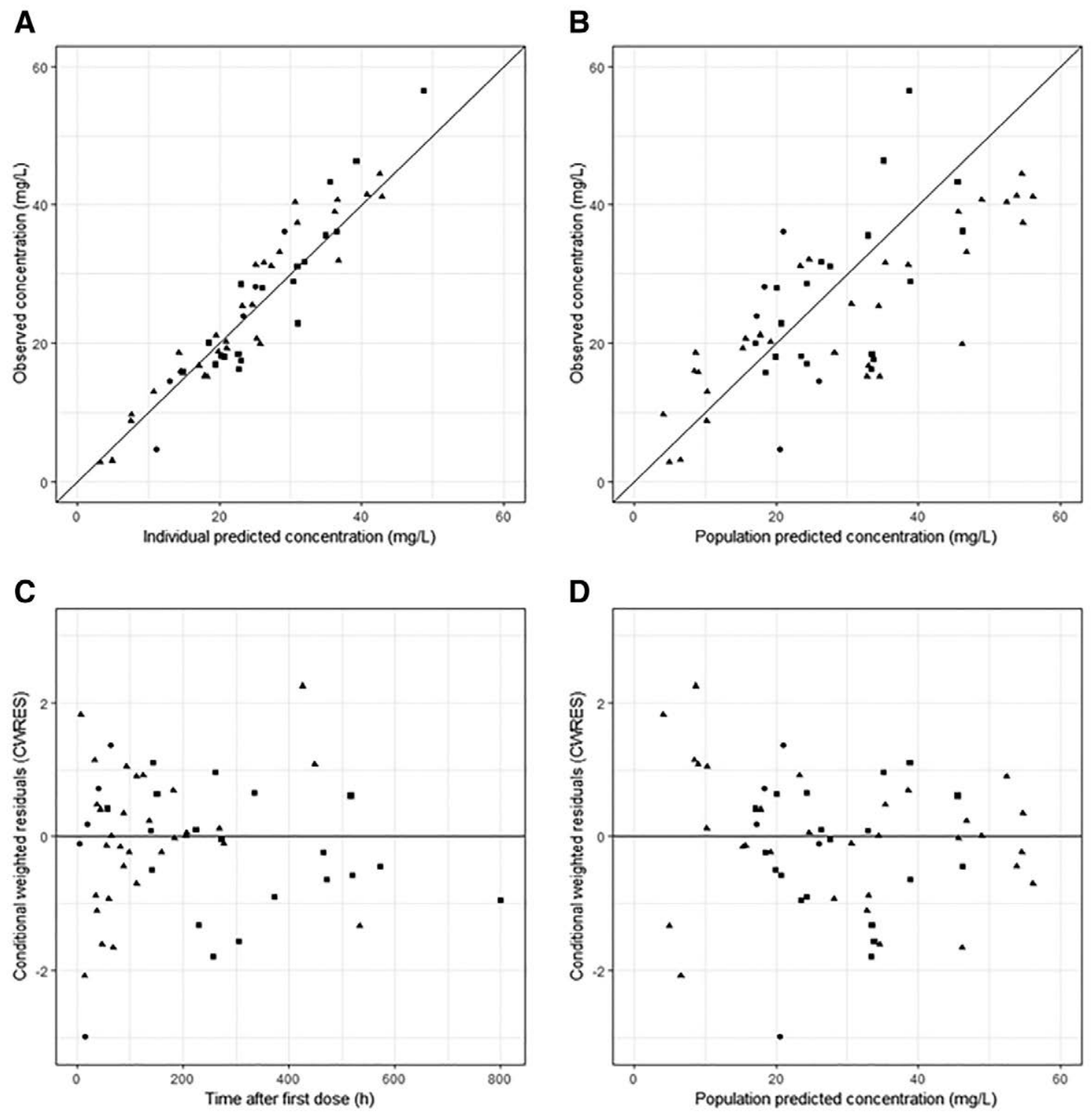

\section{ECMO}

- Before ECMO

- During ECMO

- After ECMO

Figure 2. Goodness-of-fit plots for the final model of phenobarbital pharmacokinetics in neonates on extracorporeal membrane oxygenation (ECMO). A, Population predicted phenobarbital concentration versus observed phenobarbital concentration. B, Individual predicted phenobarbital concentration versus observed phenobarbital concentration. C, Conditional weighted residuals (CWRES) versus time after dose. D, CWRES versus population predicted concentration.

expected based on maturation only, meaning that "normal" recommended dosing should be applied again after decannulation.

\section{DISCUSSION}

This is the first study using a population pharmacokinetic approach to describe the pharmacokinetics of phenobarbital in critically ill neonates undergoing ECMO. As the number of critically ill neonates requiring ECMO is small, it is difficult to include a sufficient number of patients in studies in this population; however, population modeling allows for the gain of as much information from these data as possible, as it can handle sparse and unbalanced data (19). Our analysis showed a timedependent increase of phenobarbital clearance during ECMO treatment. Additionally, we could not identify an influence of
ECMO on volume of distribution of phenobarbital. Our population pharmacokinetic study with its limited sample size represents the first step toward understanding the pharmacokinetic characteristics of phenobarbital in neonates undergoing ECMO and provides guidance for dosing strategies in these patients.

It is known that the influence of maturation, disease progression or clinical improvement, and ECMO treatment on phenobarbital pharmacokinetics cannot be differentiated from each other during prolonged ECMO treatment. Therefore, maturation functions from a previously published model in patients with an overlapping age-range that did not receive ECMO treatment (16) were included in the model. First, it was confirmed that inclusion of these maturation functions to the model led to accurate predictions of phenobarbital concentrations obtained prior to the start of ECMO and after the 

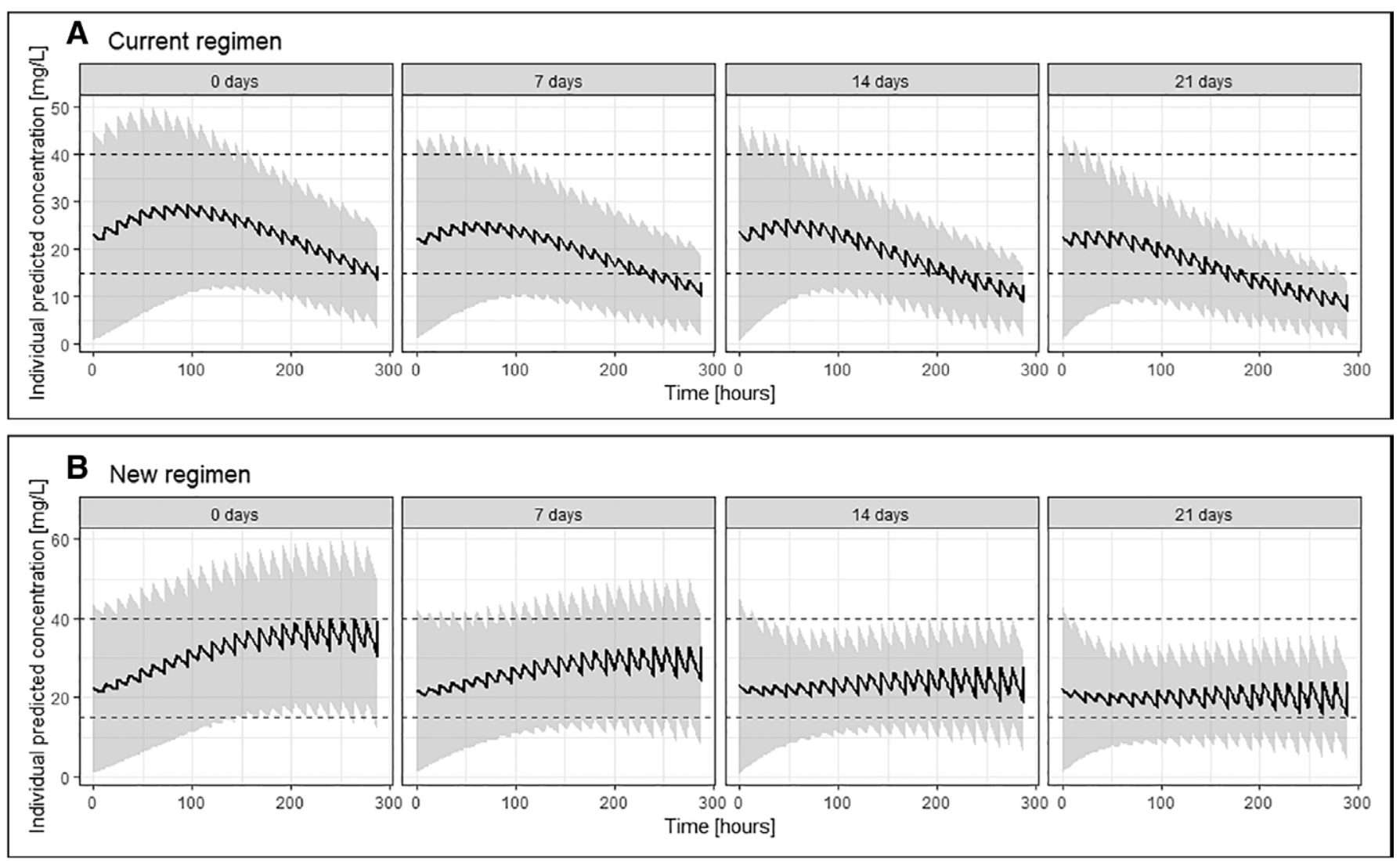

Figure 3. Simulated phenobarbital concentration over time represented as median with $95 \%$ prediction intervals, for 1,000 neonates with a birthweight of $3.21 \mathrm{~kg}$ and a postnatal age (PNA) at start of treatment of $0,7,14$, and $21 \mathrm{~d}$, with simultaneous start of extracorporeal membrane oxygenation (ECMO) and phenobarbital therapy at time $=0$. The following dosing regimens were simulated: $(\mathbf{A})$ current dosing regimen (loading dose $[\mathrm{LD}]=20 \mathrm{mg} / \mathrm{kg} ;$ maintenance dose $[M D]=5 \mathrm{mg} / \mathrm{kg} / \mathrm{d}$ for neonates of PNA $=0-14 \mathrm{~d}$ and $6 \mathrm{mg} / \mathrm{kg} / \mathrm{d}$ for neonates of PNA $=15-28 \mathrm{~d}$ divided in two daily doses); (B) new model-derived regimen ( $\mathrm{LD}=20 \mathrm{mg} / \mathrm{kg}$, start with $\mathrm{MD}=4 \mathrm{mg} / \mathrm{kg} / \mathrm{d}$ with $0.25 \mathrm{mg} / \mathrm{kg}$ increase every $12 \mathrm{hr}$ during ECMO treatment).

ECMO cessation. This indicated that the patients off ECMO and the patients from the previously published model were comparable regarding the maturational status of the pharmacokinetics of phenobarbital. Values of clearance during ECMO were found to be increased and this increase was time-dependent. Although the number of patients and obtained samples was small, diagnostics and validation of the model indicate that the data support the findings in the final model.

Apart from improved organ perfusion and oxygenation with extracorporeal support and potential clinical improvement over time, other factors may account for the increase in clearance values during ECMO. In the first place, the transit of blood through the ECMO circuit may result in degradation and/or sequestration of administered drugs (20). Sequestration of drugs in ECMO circuits is a well-known, but unpredictable phenomenon which depends on many factors. Lipophilic drugs have shown a greater tendency for drug sequestration compared with hydrophilic drugs $(6,20)$. Additionally, sequestration may increase with increasing plasma protein binding $(5,20)$. With a $\log \mathrm{P}$ of 1.47 and $25-40 \%$ protein binding one could anticipate phenobarbital sequestration to be moderate (21). Literature regarding adsorption of phenobarbital to ECMO circuit is very sparse: the only in vitro study from 1993 showed higher losses of phenobarbital in a new circuit (17\%) compared with the clinically used circuit (6\%) (22).
Clearance in our study increased linearly during ECMO, reaching more than three times increase during 12 days of ECMO. This increase is expected to eventually reach a plateau, but this was not observed during the duration of the current study. Kleiber et al (23) reported a time-dependent increase in the clearance of clonidine in a pediatric population during ECMO treatment. Clonidine is a drug with physicochemical properties similar to phenobarbital (21). The increase in clonidine clearance during ECMO was described with a sigmoidal function reaching the maximum clearance around 18-20 days after the start of ECMO treatment. Given that the duration of treatment for the individuals included in this analysis was maximum 12 days, it is possible that this was too short for the plateau in maximum clearance increase to be observed. This does imply, however, that findings in the current study should not be extrapolated beyond 12 days of ECMO treatment.

Interestingly, we found no changes in volume of distribution of phenobarbital during or after ECMO. Many previous studies showed an increase of volume of distribution for drugs during neonatal $\operatorname{ECMO}(5,24)$. There are two important factors potentially contributing to increase of volume of distribution by ECMO: the hemodilution due to circuit priming, and capillary leakage and fluid retention due to a systemic inflammatory response, either related to the patient's clinical condition or triggered by the ECMO (24). Generally, 
volume of distribution increases rapidly at the start of ECMO and then remains unchanged (24). It is possible that sparse data collection at early time points after ECMO initiation prevented the observation of changes in volume of distribution of phenobarbital in the current study.

To illustrate the implications the findings of our model have for phenobarbital dosing in neonates on ECMO, model-based simulations were performed. Median and $95 \%$ prediction interval for simulated concentrations were compared with target concentrations for neonatal seizures $(15-40 \mathrm{mg} / \mathrm{L})$, as target therapeutic concentrations for sedation and neuroprotection are still lacking (18). From the results, it seems that current recommended MD $(5 \mathrm{mg} / \mathrm{kg} / \mathrm{d}$ for neonates of PNA $=0-14 \mathrm{~d}$ and $6 \mathrm{mg} / \mathrm{kg} / \mathrm{d}$ for neonates of PNA $=15-28 \mathrm{~d}$ ) is not appropriate for older neonates and neonates being on ECMO for a longer time. The model suggests, and simulations confirm, that more optimal dosing will be achieved by a regimen that includes a MD starting with $4 \mathrm{mg} / \mathrm{kg} / \mathrm{d}$ that increases with $0.25 \mathrm{mg} / \mathrm{kg}$ every 12 hours during ECMO treatment. It should be noted that findings with our model only apply to the first 12 days of ECMO therapy, considering that the length of treatment for the patients included in this study was maximum of 12 days. Additionally, the model-derived dosing regimen assumes target concentrations obtained for neonatal seizures, while other indications may require different targets. Furthermore, the dosing guidance only takes into account adjustments based on pharmacokinetic considerations and does not take the impact of potential changes in the pharmacodynamics or safety aspects during ECMO treatment into account. Finally, as this report represents a pilot study with a limited number of samples and patients, our results need to be confirmed in future trials.

Given the large variability in pharmacokinetics of phenobarbital in neonates on ECMO (Fig. 3), still not all patients will be optimally treated by the model-derived regimen as indicated by parts of the $95 \%$ prediction interval of simulated concentrations being outside of the therapeutic range. Therefore, TDM should still be considered in individual cases, even with the model-derived regimen. Monitoring of the plasma concentrations should also be repeated over time, as the simulations show that patients that once had adequate phenobarbital exposure could at later time points get to overexposure or underexposure. This especially applies for the neonates in which ECMO is initiated on the first day of life. Finally, we would like to stress that population pharmacokinetic models are limited to the drug and patient population used in the analysis. Therefore, new analyses would be required for new drugs. The ability of population models to deal with sparse and unbalanced data would make this approach ideally suitable for such future analyses.

\section{CONCLUSIONS}

Our results indicate that that the current recommended phenobarbital MD may not be appropriate for neonates of PNA greater than 7 days being on ECMO for at least 8 to 12 days. Time-dependent increase in phenobarbital clearance results in continuously decreasing phenobarbital exposure in patients during ECMO treatment and will thus increase the risk of therapeutic failure over time. This implies that continuously increasing doses of phenobarbital over time are needed for these patients. Due to high remaining unexplained variability, repeated TDM over time should still be considered.

Supplemental digital content is available for this article. Direct URL citations appear in the printed text and are provided in the HTML and PDF versions of this article on the journal's website (http://journals.lww.com/ pccmjournal).

This work was supported by the Charles University Project Progress Q25 and by Ministry of Health, Czech Republic - conceptual development of research organization ("General University Hospital") MH CZ-DRO VFN64165.

Dr. Krekels disclosed off-label product use of phenobarbital in neonates on extracorporeal membrane oxygenation. The remaining authors have disclosed that they do not have any potential conflicts of interest.

For information regarding this article, E-mail: martta@gmail.com

\section{REFERENCES}

1. Wildschut ED, Ahsman MJ, Houmes RJ, et al: Pharmacotherapy in neonatal and pediatric extracorporeal membrane oxygenation (ECMO). Curr Drug Metab 2012; 13:767-777

2. Lorusso R, Alexander P, Rycus P, et al: The Extracorporeal Life Support Organization Registry: Update and perspectives. Ann Cardiothorac Surg 2019; 8:93-98

3. Wildschut ED, van Saet A, Pokorna P, et al: The impact of extracorporeal life support and hypothermia on drug disposition in critically ill infants and children. Pediatr Clin North Am 2012; 59:1183-1204

4. Wildschut ED, Ahsman MJ, Allegaert K, et al: Determinants of drug absorption in different ECMO circuits. Intensive Care Med 2010; 36:2109-2116

5. Ha MA, Sieg AC: Evaluation of altered drug pharmacokinetics in critically ill adults receiving extracorporeal membrane oxygenation. Pharmacotherapy 2017; 37:221-235

6. Shekar K, Fraser JF, Smith MT, et al: Pharmacokinetic changes in patients receiving extracorporeal membrane oxygenation. J Crit Care 2012; 27:741.e9-e18

7. Cheng V, Abdul-Aziz MH, Roberts JA, et al: Optimising drug dosing in patients receiving extracorporeal membrane oxygenation. $J$ Thorac Dis 2018; 10:S629-S641

8. Italiano D, Capuano A, Alibrandi A, et al: Indications of newer and older anti-epileptic drug use: Findings from a southern Italian general practice setting from 2005-2011. Br J Clin Pharmacol 2015; 79:1010-1019

9. Šíma M, Pokorná P, Hartinger J, et al: Estimation of initial phenobarbital dosing in term neonates with moderate-to-severe hypoxic ischaemic encephalopathy following perinatal asphyxia. J Clin Pharm Ther 2018; 43:196-201

10. Šíma $M$, Pokorná $P$, Hronová $K$, et al: Effect of co-medication on the pharmacokinetic parameters of phenobarbital in asphyxiated newborns. Physiol Res 2015; 64:S513-S519

11. Fischer JH, Lockman LA, Zaske D, et al: Phenobarbital maintenance dose requirements in treating neonatal seizures. Neurology 1981; 31:1042-1044

12. Jolley ME, Stroupe SD, Schwenzer KS, et al: Fluorescence polarization immunoassay. iii. An automated system for therapeutic drug determination. Clin Chem 1981; 27:1575-1579

13. Lindbom L, Pihlgren $P$, Jonsson EN, et al: PsN-Toolkit-a collection of computer intensive statistical methods for non-linear mixed effect modeling using NONMEM. Comput Methods Programs Biomed 2005; 79:241-257

14. Lindbom L, Ribbing J, Jonsson EN: Perl-speaks-NONMEM (PsN)-a Perl module for NONMEM related programming. Comput Methods Programs Biomed 2004; 75:85-94 
15. Keizer RJ, van Benten M, Beijnen JH, et al: Piraña and PCluster: A modeling environment and cluster infrastructure for NONMEM. Comput Methods Programs Biomed 2011; 101:72-79

16. Völler S, Flint RB, Stolk LM, et al; DINO study group: Model-based clinical dose optimization for phenobarbital in neonates: An illustration of the importance of data sharing and external validation. Eur J Pharm Sci 2017; 109S:S90-S97

17. Comets E, Brendel K, Mentré F: Computing normalised prediction distribution errors to evaluate nonlinear mixed-effect models: The npde add-on package for R. Comput Methods Programs Biomed 2008; 90:154-166

18. Formulary DNCs. 2019. Available at: https://www.kinderformularium. $\mathrm{nl} /$. Accessed April 19, 2020

19. De Cock RF, Piana C, Krekels EH, et al: The role of population PK-PD modelling in paediatric clinical research. Eur J Clin Pharmacol 2011; 67(Suppl 1):5-16
20. Shekar K, Roberts JA, Mcdonald Cl, et al: Protein-bound drugs are prone to sequestration in the extracorporeal membrane oxygenation circuit: Results from an ex vivo study. Crit Care 2015; 19:164

21. Wishart DS, Feunang YD, Guo AC, et al: DrugBank 5.0: A major update to the DrugBank database for 2018. Nucleic Acids Res 2018; 46:D1074-D1082

22. Dagan O, Klein J, Gruenwald C, et al: Preliminary studies of the effects of extracorporeal membrane oxygenator on the disposition of common pediatric drugs. Ther Drug Monit 1993; 15:263-266

23. Kleiber N, Mathôt RAA, Ahsman MJ, et al: Population pharmacokinetics of intravenous clonidine for sedation during paediatric extracorporeal membrane oxygenation and continuous venovenous hemofiltration. Br J Clin Pharmacol 2017; 83:1227-1239

24. Di Nardo M, Wildschut ED: Drugs pharmacokinetics during venovenous extracorporeal membrane oxygenation in pediatrics. $J$ Thorac Dis 2018; 10:S642-S652 